\title{
Rank reduction and bordered inversion
}

\author{
Aurél Galántai
}




\title{
RANK REDUCTION AND BORDERED INVERSION
}

\author{
Aurél Galántai \\ Institute of Mathematics, University of Miskolc \\ 3515 Miskolc - Egyetemváros, Hungary \\ matgal@gold.uni-miskolc.hu
}

[Received October 8, 2001]

\begin{abstract}
We clarify the connection between the rank reduction algorithm and the bordered inversion method and their related sequences.

Mathematical Subject Classification: 15A09, 15A21, 15A23, 65F30, 65 F99.

Keywords: Rank reduction, bordered inversion, inverse factorization, conjugation
\end{abstract}

\section{Introduction}

The rank reduction algorithm and the bordered inversion method play important roles in numerical linear algebra. The rank reduction algorithm, while keeping the size of the matrix, decreases its rank to zero. The bordered inversion method, starting from a small size leading principal submatrix, increases the size and rank of the submatrix to obtain an inverse. The formulae of the two methods show certain formal similarities. In this paper we are looking for a deeper connection between the two methods. Using unexplored observations of Egerváry [4], we determine three kinds of relationship between the two algorithms and associated sequences. These results are essentially the following. The rank reduction operation gives the reverse bordered inversion formula. Using the inverse LDU decomposition algorithm included in the bordered inversion method, we can produce the same full rank factorization as the rank reduction algorithm. Finally, the bordered inversion formula leads to new multiplicative canonical forms of the rank reduction algorithm.

\section{Preliminary results}

We need the following results. For proofs and other details, see Ouellette [12].

Lemma 1 (Guttman-1) Let

$$
A=\left[\begin{array}{cc}
E & F \\
G & H
\end{array}\right] \in R^{m \times n} \quad\left(E \in R^{k \times k}\right) .
$$

If $E$ is nonsingular, then

$$
\operatorname{rank}(A)=\operatorname{rank}(E)+\operatorname{rank}\left(H-G E^{-1} F\right) .
$$


Corollary 2 If $A$ and $E$ are nonsingular, then the Schur complement

$$
S=(A / E)=H-G E^{-1} F
$$

is also nonsingular.

Lemma 3 (Guttman-2) Let

$$
A=\left[\begin{array}{cc}
E & F \\
G & H
\end{array}\right] \in R^{m \times n} \quad\left(H \in R^{j \times j}\right) .
$$

If $H$ is nonsingular, then

$$
\operatorname{rank}(A)=\operatorname{rank}(H)+\operatorname{rank}\left(E-F H^{-1} G\right) .
$$

Corollary 4 If $A$ and $H$ are nonsingular, then the Schur complement

$$
T=(A / H)=E-F H^{-1} G
$$

is also nonsingular.

\section{The inverse of partitioned matrices}

Here we recall the most important results concerning the inverse of partitioned matrices. For proofs and related results see Ouellette [12]. Let

$$
A=\left[\begin{array}{cc}
E & F \\
G & H
\end{array}\right] \in R^{n \times n} \quad\left(E \in R^{k \times k}\right)
$$

and $E$ be nonsingular.

Theorem 5 (Banachiewicz-Frazer-Duncan-Collar) If the nonsingular matrix $A \in$ $R^{n \times n}$ is partitioned in the form (3.1), where $E$ has an inverse, then $A^{-1}$ can be expressed in the partitioned form

$$
A^{-1}=\left[\begin{array}{cc}
E^{-1}+E^{-1} F S^{-1} G E^{-1} & -E^{-1} F S^{-1} \\
-S^{-1} G E^{-1} & S^{-1}
\end{array}\right] .
$$

Remark 6 Provided that $E$ is invertible, $A$ is invertible if and only if $S$ is invertible.

Remark 7 Formula (3.2) can be written in the following form

$$
A^{-1}=\left[\begin{array}{cc}
E^{-1} & 0 \\
0 & 0
\end{array}\right]+\left[\begin{array}{c}
E^{-1} F \\
-I
\end{array}\right] S^{-1}\left[G E^{-1},-I\right] .
$$




\section{The bordered inversion method}

Formula (3.2) is the basis for the bordered inversion or escalator method of Frazer, Duncan and Collar [6]. For $k=1, \ldots, m$ let

$$
E_{k+1}=\left[\begin{array}{cc}
E_{k} & F_{k} \\
G_{k} & H_{k}
\end{array}\right] \quad\left(E_{k} \in R^{n_{k} \times n_{k}}, F_{k}, G_{k}^{T} \in R^{n_{k} \times p_{k}}, H_{k} \in R^{p_{k} \times p_{k}}\right) .
$$

Hence $n_{k+1}=n_{k}+p_{k}$ and $E_{m+1}=E$. If $E_{k}$ and $S_{k}=H_{k}-G_{k} E_{k}^{-1} F_{k}$ are nonsingular, then $E_{k+1}$ is also nonsingular and by Theorem 5 we can calculate $E_{k+1}^{-1}$.

\section{The block bordered inversion algorithm}

1. Determine $E_{1}^{-1}$.

2. For $k=1, \ldots, m$ set

$$
S_{k}=H_{k}-G_{k} E_{k}^{-1} F_{k}
$$

and

$$
E_{k+1}^{-1}=\left[\begin{array}{cc}
E_{k}^{-1}+E_{k}^{-1} F_{k} S_{k}^{-1} G_{k} E_{k}^{-1} & -E_{k}^{-1} F_{k} S_{k}^{-1} \\
-S_{k}^{-1} G_{k} E_{k}^{-1} & S_{k}^{-1}
\end{array}\right]
$$

end

It is clear that $E_{m+1}^{-1}=E^{-1}$ provided that $S_{k}$ is nonsingular for $k=1, \ldots, m$. If $S_{k}$ is singular for an index $k$, then the Guttman lemma implies that $E_{k+1}$ is singular.

Theorem 8 (Guttman) Let $E$ be nonsingular. The block bordered inversion method gives the inverse of $E$ if and only if all $E_{k}$ are nonsingular for $k=1, \ldots, m$.

Guttman suggested several block variants of the bordered inversion method. For details, see Guttman [10] and Ouellette [12]. We also note that Gergely [9] observed the equivalence of the bordered inversion method with the Gauss-Jordan inversion.

\section{The rank reduction algorithm}

The rank reduction procedure of Egerváry is based on the following result.

Theorem 9 (Egerváry-Guttman-Wedderburn) Let $H, S \in R^{m \times n}$ and $\operatorname{rank}(H) \geq$ $\operatorname{rank}(S)=k$. Furthermore let $U R^{-1} V^{T}\left(U \in R^{m \times k}, R \in R^{k \times k}, V \in R^{n \times k}\right)$ be a full rank factorization of $S$. Then

$$
\operatorname{rank}(H-S)=\operatorname{rank}(H)-\operatorname{rank}(S),
$$

if and only if

$$
U=H X, \quad V^{T}=Y^{T} H, \quad Y^{T} H X=R
$$

for some matrices $X \in R^{n \times k}$ and $Y \in R^{m \times k}$. 
Remark 10 We can write that

$$
\operatorname{rank}(H-S)=\operatorname{rank}(H)-\operatorname{rank}(S) \Leftrightarrow S=H X\left(Y^{T} H X\right)^{-1} Y^{T} H .
$$

For the proof of the theorem see Egerváry [4], Guttman [11], Cline and Funderlic [3], Ouellette [12] and Galántai [7].

The rank reduction algorithm which is important in full rank factorizations and conjugation has the following form.

Let $A_{1}=A, X_{i} \in R^{n \times l_{i}}, Y_{i} \in R^{m \times l_{i}}, l_{i} \geq 1$ and $Y_{i}^{T} A_{i} X_{i}$ be nonsingular for $i=1,2, \ldots, k$.

\section{The rank reduction procedure}

$$
A_{i+1}=A_{i}-A_{i} X_{i}\left(Y_{i}^{T} A_{i} X_{i}\right)^{-1} Y_{i}^{T} A_{i} \quad(i=1,2, \ldots, k),
$$

where $\operatorname{rank}(A) \geq \sum_{i=1}^{k} l_{i}$.

The algorithm is said to be breakdown free, if all $Y_{i}^{T} A_{i} X_{i}$ are nonsingular for $i=1,2, \ldots, k$. Assume that

$$
\operatorname{rank}(A)=\sum_{i=1}^{k} l_{i}
$$

In this case, $A_{k+1}=0$. Let

$$
X=\left[X_{1}, \ldots, X_{k}\right], \quad Y=\left[Y_{1}, \ldots, Y_{k}\right]
$$

and

$$
X^{(i)}=\left[X_{1}, \ldots, X_{i}\right], \quad Y^{(i)}=\left[Y_{1}, \ldots, Y_{i}\right]
$$

Then the following theorem is true.

Theorem 11 The rank reduction procedure can be carried out breakdown free if and only if $Y^{T} A X=\left[Y_{i}^{T} A X_{j}\right]_{i, j=1}^{k}$ is block strongly nonsingular ${ }^{1}$. In this event the rank reduction procedure has the canonical form

$$
A_{i+1}=A-A X^{(i)}\left(Y^{(i) T} A X^{(i)}\right)^{-1} Y^{(i) T} A \quad(i=1, \ldots, k) .
$$

For proof, see Galántai [7], [8]. For $i=k$ the relation

$$
A=A X\left(Y^{T} A X\right)^{-1} Y^{T} A
$$

holds, where $Z=X\left(Y^{T} A X\right)^{-1} Y^{T}$ is a reflexive inverse of $A$. It is also easy to see that algorithm (5.3) leads to the full rank factorization

$$
A=Q D^{-1} P^{T},
$$

\footnotetext{
${ }^{1}$ It means that the matrix has a block $L U$ decomposition without pivoting.
} 
where

$$
P=\left[A_{1}^{T} Y_{1}, \ldots, A_{k}^{T} Y_{k}\right], \quad Q=\left[A_{1} X_{1}, \ldots, A_{k}^{T} X_{k}\right]
$$

and

$$
D=\operatorname{diag}\left(Y_{1}^{T} A_{1} X_{1}, \ldots, Y_{k}^{T} A_{k} X_{k}\right) .
$$

If a square matrix $B$ has a block LDU decomposition with respect to a given partition, then this unique factorization will be denoted by $B=L_{B} D_{B} U_{B}$. Then we can prove

Theorem 12 Let $Y^{T} A X$ be block strongly nonsingular. The components of the full rank factorization (5.5) are

$$
P=A^{T} Y L_{Y^{T} A X}^{-T}, \quad Q=A X U_{Y^{T} A X}^{-1}, \quad D=D_{Y^{T} A X},
$$

and $A$ can be expressed in the form

$$
A=\left(A X U_{Y^{T} A X}^{-1}\right) D_{Y^{T} A X}^{-1}\left(L_{Y^{T} A X}^{-1} Y^{T} A\right) .
$$

For proof, see Galántai [7], [8].

\section{Rank-reduction and bordered inversion I}

We show a surprisingly simple connection between the rank reduction operation and the bordered inversion.

Theorem 13 Assume that

$$
A=\left[\begin{array}{cc}
E & F \\
G & H
\end{array}\right] \in R^{n \times n} \quad\left(E \in R^{k \times k}\right)
$$

and

$$
J=\left[\begin{array}{l}
0 \\
I_{n-k}
\end{array}\right] \in R^{n \times(n-k)} .
$$

Then

$$
A-A J\left(J^{T} A J\right)^{-1} J^{T} A=\left[\begin{array}{cc}
(A / H) & 0 \\
0 & 0
\end{array}\right],
$$

provided that $H$ is invertible ${ }^{2}$.

Proof. By simple calculation we have

$$
A J\left(J^{T} A J\right)^{-1} J^{T} A=\left[\begin{array}{c}
F \\
H
\end{array}\right] H^{-1}[G, H]=\left[\begin{array}{cc}
F H^{-1} G & F \\
G & H
\end{array}\right]
$$

and

$$
A-J\left(J^{T} A J\right)^{-1} J^{T} A=\left[\begin{array}{cc}
E-F H^{-1} G & 0 \\
0 & 0
\end{array}\right],
$$

which was to be proved.

\footnotetext{
${ }^{2}$ This condition is in agreement with the strong block nonsingularity of $Y^{T} A X$. In fact, $X_{1}=$ $Y_{1}=J$ and $Y_{1}^{T} A X_{1}=H$.
} 
Remark 14 If $J$ is replaced by $\widehat{J}=\left[\begin{array}{c}I_{k} \\ 0\end{array}\right]$, then we have

$$
A-A \widehat{J}\left(\widehat{J}^{T} A \widehat{J}\right)^{-1} \widehat{J}^{T} A=\left[\begin{array}{cc}
0 & 0 \\
0 & (A / E)
\end{array}\right],
$$

provided that $E$ is nonsingular.

Remark 15 If $A$ and $H$ are replaced by $A^{-1}$ and $S^{-1}$, respectively then we have $\left(A^{-1} / S^{-1}\right)=E^{-1}$. This means that

$$
A^{-1}-A^{-1} J\left(J^{T} A^{-1} J\right)^{-1} J^{T} A^{-1}=\left[\begin{array}{cc}
E^{-1} & 0 \\
0 & 0
\end{array}\right] \text {. }
$$

We just obtained that one rank reduction step on matrix $A^{-1}$ results in the inverse of the leading principal submatrix $E$. Relation (6.3) gives a direct connection between the rank reduction and the bordered inversion. It was first discovered by Egerváry [4] in the scalar case $(H \in R)$, who developed it for solving difference equations with modified boundary conditions [5]. The same idea appears in Brezinski et al. [2], who give credit to Duncan and call it reverse bordered inversion. Their variant of identity (6.3) is obtained in the following way.

Let

$$
A^{-1}=\left[\begin{array}{cc}
\widehat{E} & \widehat{F} \\
\widehat{G} & \widehat{H}
\end{array}\right] \text {. }
$$

Then

$$
\begin{gathered}
\widehat{E}=E^{-1}+E^{-1} F S^{-1} G E^{-1}, \quad \widehat{F}=-E^{-1} F S^{-1}, \\
\widehat{G}=-S^{-1} G E^{-1}, \quad \widehat{H}=S^{-1} .
\end{gathered}
$$

As $\widehat{F}(\widehat{H})^{-1}=-E^{-1} F$ and $\widehat{F}(\widehat{H})^{-1} \widehat{G}=E^{-1} F S^{-1} G E^{-1}$ we have

$$
E^{-1}=\widehat{E}-E^{-1} F S^{-1} G E^{-1}=\widehat{E}-\widehat{F}(\widehat{H})^{-1} \widehat{G},
$$

which is exactly the reverse bordered inversion formula of Brezinski et al. [2].

\section{The bordered inversion method and triangular factorization}

We associate sequences with the bordered inversion algorithm which produce the inverse LDU factorizations of the leading principal submatrices $E_{k}$. It is based on the following observation. Let $E=L D U$ be the unique block LDU decomposition of $E$. If $E$ is not partitioned, then we just take $L=U=I$ and $D=E$. If $A$ has the form (3.1), then the inverse formula (3.3) can be written as

$$
\begin{aligned}
A^{-1} & =\left[\begin{array}{cc}
U^{-1} & -E^{-1} F \\
0 & I
\end{array}\right]\left[\begin{array}{cc}
D^{-1} & 0 \\
0 & S^{-1}
\end{array}\right]\left[\begin{array}{cc}
L^{-1} & 0 \\
-G E^{-1} & I
\end{array}\right] \\
& =U_{A}^{-1} D_{A}^{-1} L_{A}^{-1}
\end{aligned}
$$


which is the inverse block LDU decomposition of $A^{-1}$. Thus we can define the following sequences related to the bordered inversion algorithm of Section 4.

Let $E_{k}=L_{k} D_{k} U_{k}$ denote the unique LDU decomposition of $E_{k}$ for $k=1, \ldots, m$.

\section{Inverse $L D U$ decomposition algorithm}

For $k=1, \ldots, m$ set

$$
\begin{gathered}
L_{k+1}^{-1}=\left[\begin{array}{cc}
L_{k}^{-1} & 0 \\
-G_{k} E_{k}^{-1} & I
\end{array}\right], \quad D_{k+1}^{-1}=\left[\begin{array}{cc}
D_{k}^{-1} & 0 \\
0 & S_{k}^{-1}
\end{array}\right], \\
U_{k+1}^{-1}=\left[\begin{array}{cc}
U_{k}^{-1} & -E_{k}^{-1} F_{k} \\
0 & I
\end{array}\right] .
\end{gathered}
$$

end

It is clear that $E_{k+1}^{-1}=U_{k+1}^{-1} D_{k+1}^{-1} L_{k+1}^{-1}$ and

$$
E^{-1}=U_{m+1}^{-1} D_{m+1}^{-1} L_{m+1}^{-1} \text {. }
$$

The above algorithm, which requires no extra operations, is based on a remark by Egerváry [4], according to which the bordered inversion (or escalator) method "furnishes the triangular factorisation of the inverse".

The associated procedure can be carried out if and only if the bordered inversion can be so. Hence the necessary and sufficient condition for performing the algorithm without breakdown is the block strong nonsingularity of $E$.

\section{Rank-reduction and bordered inversion II}

Here we determine a new relationship between rank reduction and bordered inversion. More precisely, we show that certain sequences related to the rank reduction and the bordered inversion algorithms, respectively, can be tied together. We recall the facts that

1. The associated sequences of the bordered inversion method produce $U_{E}^{-1}, D_{E}^{-1}$ and $L_{E}^{-1}$.

2. The rank reduction method produces the full rank factorization

$$
A=Q D^{-1} P^{T}=\left(A X U_{Y^{T} A X}^{-1}\right) D_{Y^{T} A X}^{-1}\left(L_{Y^{T} A X}^{-1} Y^{T} A\right) .
$$

If $X=B^{T} V$, then the pair $(P, V)$ is block $B$-conjugate (see Galántai [7], [8]). This conjugation property plays an important role in the ABS methods (see AbaffySpedicato [1]).

Having the two observations above, we can easily derive the following

\section{Conjugation algorithm}

1. Apply the inverse $L D U$ decomposition algorithm to the matrix $Y^{T} A X$ and get $L_{Y^{T} A X}^{-1} \cdot$ 
2. Set

$$
P=A^{T} Y L_{Y^{T}}^{-T} A X
$$

end

Thus we have a new connection between rank reduction and bordered inversion. It is also clear that, similarly, we can have $Q$ and $D^{-1}$. Finally we mention that Stewart [13] gave a conjugation algorithm which is based on LU factorization.

\section{Rank-reduction and the reverse bordered inversion}

We deal with the last connection found, which is, in fact, an application of identity (6.3) to obtain new canonical forms for $A_{i+1}$. We start with the formula (5.4), which tells us that

$$
A_{i+1}=A-A X^{(i)}\left(Y^{(i) T} A X^{(i)}\right)^{-1} Y^{(i) T} A \quad(i=1, \ldots, k) .
$$

Let $I^{(j)}$ be the first $j$ columns of the identity matrix $I_{n}$. Furthermore let $\omega_{i}=\sum_{j=1}^{i} l_{j}$. Then $X^{(i)}=X I^{\left(\omega_{i}\right)}$ and $Y^{(i)}=Y I^{\left(\omega_{i}\right)}$ and we can write

$$
A_{i+1}=A X\left[\left(Y^{T} A X\right)^{-1}-I^{\left(\omega_{i}\right)}\left(Y^{(i) T} A X^{(i)}\right)^{-1} I^{\left(\omega_{i}\right) T}\right] Y^{T} A .
$$

As

$$
I^{\left(\omega_{i}\right)}\left(Y^{(i) T} A X^{(i)}\right)^{-1} I^{\left(\omega_{i}\right) T}=\left[\begin{array}{cc}
\left(Y^{(i) T} A X^{(i)}\right)^{-1} & 0 \\
0 & 0
\end{array}\right]
$$

we have the following

Theorem $16 A_{i+1}$ has the following canonical form

$$
A_{i+1}=A X\left(\left(Y^{T} A X\right)^{-1}-\left[\begin{array}{cc}
\left(Y^{(i) T} A X^{(i)}\right)^{-1} & 0 \\
0 & 0
\end{array}\right]\right) Y^{T} A
$$

under the conditions of Theorem 11.

We can observe that in the parentheses we have a variant of the identity (6.3) with $Y^{T} A X$ instead of $A^{-1}$. Thus

$$
\begin{aligned}
& \left(Y^{T} A X\right)^{-1}-\left[\begin{array}{cc}
\left(Y^{(i) T} A X^{(i)}\right)^{-1} & 0 \\
0 & 0
\end{array}\right] \\
& =\left(Y^{T} A X\right)^{-1} J\left[J^{T}\left(Y^{T} A X\right)^{-1} J\right]^{-1} J^{T}\left(Y^{T} A X\right)^{-1},
\end{aligned}
$$

where

$$
J=\left[\begin{array}{c}
0 \\
I_{n-\omega_{i}}
\end{array}\right]
$$

Hence by simple calculations we have 
Theorem $17 A_{i+1}$ has the following canonical form

$$
A_{i+1}=Y^{-T} J\left(J^{T} X^{-1} A^{-1} Y^{-T} J\right)^{-1} J^{T} X^{-1}
$$

under the conditions of Theorem 11.

Assuming that

$$
\left(Y^{T} A X\right)^{-1}=\left[\begin{array}{cc}
\widehat{E} & \widehat{F} \\
\widehat{G} & \widehat{H}
\end{array}\right]
$$

we can obtain a third form of $A_{i+1}$.

Theorem $18 A_{i+1}$ has the following canonical form

$$
A_{i+1}=Y^{-T}\left[\begin{array}{cc}
0 & 0 \\
0 & \widehat{H}^{-1}
\end{array}\right] X^{-1}
$$

under the conditions of Theorem 11.

These new multiplicative canonical forms indicate different properties of the rank reduction algorithm. The first canonical form indicates the decrease of $\left\|A_{k+1}\right\|$. The second form shows the projector properties of the rank reduction matrix $A_{i+1}$. The last canonical form shows the structure of $A_{k+1}$ in terms of inverse quantities. These three new forms certainly give a better understanding of rank reduction and may lead to further new results.

Acknowledgement: This work was supported by the CNR International short-term mobility program, Year 2001.

\section{REFERENCES}

[1] Abaffy J. and Spedicato, E.: ABS-projection Algorithms: Mathematical Techniques for Linear and Nonlinear Algebraic Equations, Ellis Horwood, Chichester, 1989.

[2] Brezinski, C., Morandi Cecchi, M. and Redivo-Zaglia, M.: The reverse bordering method, SIAM J. Matrix. Anal. Appl., 15, (1994\}, 922-937.

[3] Cline, R.E. and Funderlic, R.E.: The rank of a difference of matrices and associated generalized inverses, Lin. Alg. Appl., 24, (1979), 185-215.

[4] EGerVÁRY, E.: On rank-diminishing operations and their applications to the solution of linear equations, ZAMP, XI., (1960), 376-386.

[5] Egerváry, J.: Über eine Methode zur numerischen Lösung der Poissonschen Differenzengleichung für beliebige Gebiete, Acta Mathematica Academiae Scientiarium Hungaricae, 11, (1960), 341-361.

[6] Frazer, A., Duncan, J., Collar, R.: Elementary matrices, Cambridge University Press, 1938.

[7] Galántai A.: Rank reduction and conjugation, Mathematical Notes, Miskolc, 1(1), (2000), 11-33. 
[8] Galántai A.: Rank reduction, factorization and conjugation, Linear and Multilinear Algebra, Vol. 49, (2001), 195-207.

[9] Gergely J.: Mátrixinvertáló módszerekröl (On matrix inversion methods, in Hungarian), Alkalmazott Matematikai Lapok, 2, (1976), 359-362.

[10] Guttman, L.: Enlargement methods for computing the inverse matrix, Ann. Math. Statist., 17, (1946), 336-343.

[11] Guttman, L.: A necessary and sufficient formula for matrix factoring, Psychometrika, 22, (1957), 79-91.

[12] Ouellette, D.V.: Schur complements and statistics, Lin. Alg. Appl., 36, (1981), 187-295.

[13] Stewart, G.W.: Conjugate direction methods for solving systems of linear equations, Numerische Mathematik, 21, (1973), 285-297. 